\title{
Switched $\mathcal{H}_{\infty}$ Detection Filter Design for Nonlinear Systems
}

\author{
Zsolt Horváth $^{1}$, András Edelmayer ${ }^{2,3}$ \\ ${ }^{1}$ School of Postgraduate Studies of Multidisciplinary Sciences, Faculty of Technical Sciences, Széchenyi István University, Győr, Hungary \\ ${ }^{2}$ Department of Informatics Engineering, Faculty of Technical Sciences, Széchenyi István University, Győr, Hungary \\ ${ }^{3}$ Systems and Control Laboratory, Institute for Computer Science and Control, Hungarian Academy of Sciences, Budapest, Hungary
}

\section{Email address:}

zsolt2.horvath@audi.hu (Z. Horváth), edelmayer@sze.hu (A. Edelmayer), edelmayer@sztaki.mta.hu (A. Edelmayer)

\section{To cite this article:}

Zsolt Horváth, András Edelmayer. Switched $\mathcal{H}_{\infty}$ Detection Filter Design for Nonlinear Systems. American Journal of Mechanical and Industrial Engineering. Vol. 4, No. 1, 2019, pp. 1-10. doi: 10.11648/j.ajmie.20190401.11

Received: February 26, 2019; Accepted: April 3, 2019; Published: May 7, 2019

\begin{abstract}
This paper presents the application of the concept of $\mathcal{H}_{\infty}$ detection filters to the detection of faults in nonlinear systems. The nonlinear dynamics in specific meaningful points of the operation is approximated by means of a matched array of linear systems. Then, linear $\mathcal{H}_{\infty}$ filters are designed for each particular subsystem and a switching scheme is applied to carefully choose the most suitable filter regarding the operational characteristics of the plant in real time. Stability of the switching process is guaranteed by keeping the switching time between two consecutive switching large enough to ensure a proper falloff of filter transients. Therefore, apart from the solution of the standard linear-quadratic optimization problem represented by the $\mathcal{H}_{\infty}$ detection filter design problem one has to derive sufficient conditions for the observation error dynamics to be globally asymptotically stable during switching. The goal is to find a common minimum of the switching time to each specific $\mathcal{H}_{\infty}$ level calculated separately for every single filter that can be used as a restriction for the switching signal. The idea is demonstrated with the application to the detection of faults in the air path of a diesel engine. The results can be considered as the extension of the standard linear $\mathcal{H}_{\infty}$ fault detection filtering problem to nonlinear systems.
\end{abstract}

Keywords: Switched Linear System, Dwell Time, Switched $\mathcal{H}_{\infty}$ Fault Detection Filter, MFARE

\section{Introduction}

The design of detection filters for nonlinear systems is a mature field of engineering. Therefore, in the past two decades a wide range of different methods have been investigated, also specially in the field of diagnostics of the combustion engines. A nonlinear unknown input observer (NUIO) for detection of actuator faults in diesel engines is presented. [1] For similar case another research proposes the usage of neural network for the filter implementation. [2] To comply with the severe requirements on recursion speed of the filter by using nonlinear models a fuzzy filtering approach was proposed for sensor fault detection and isolation in the diesel air path. [3] A fault detection in combustion engines using nonlinear parity equations is applied. [4] Geometric LPV (Linear Parameter Variable) fault detection filter for commercial aircrafts is designed. [5] A detection filter is applied for nonlinear systems by means of geometric view on inversion-based model. [6]

Generally, in order to make the nonlinear problem formulations tractable, some form of model reduction, approximation and/or process simplification is often inevitable to tackle computational burden and satisfy eligibility requirements for implementation. Linearization and the reduction of the order of the dynamics are the most frequently used techniques for the reduction of complexity of physical processes normally represented by nonlinear system models. The main drawback of linearization is that in certain operating points (i.e., where the linearization was done) one may get big deviations from the ideal operating conditions making the approximation of linearization highly inaccurate that compromises the performance of the filter. Therefore, relying on a linear modeling approach may result in a completely useless filter design approach in demanding applications.

In this paper a $\mathcal{H}_{\infty}$ detection filter design approach is proposed which is based on the concept of switched linear 
systems. This includes the linearization of the plant in a number of equilibrium points and the design of a set of linear filters, one separate filter for each linearized subsystem. Then, a selection mechanism is applied to carefully choose the most suitable filter regarding the operational characteristics of the plant in real time. Thus, the nonlinear detection problem in specific meaningful points of the operation is approximated by means of a matched array of linear filters. This idea can be accomplished by means of the application of two solution methods: i) switching or ii) interpolation.

Application of the idea of switching systems for nonlinear control have been extensively studied in earlier works. As a result, many useful results are now available. [7-11] A detailed survey on the theory of switched linear systems can be found in the references. [12-13] A common problem posed by most of the work was to ensure controller (filter) stability during the switching process. As it was stated by several authors. The asymptotic stability of the solution can be ensured when we switch between the subsystems slowly enough. To be more precise, when the intervals between two consecutive switching, which is called dwell time, are large enough. [7, 10, 13]

In the following sections we propose a $\mathcal{H}_{\infty}$ filter design strategy where special attention is given to the stability of the estimation error of the detection when replacing a filter with another.

When specifying a $\mathcal{H}_{\infty}$ fault detection filter the robustness is ensured by the application of a design trade-off between the worst-case disturbance and the L2 -norm of the filter error. This method requires the solution of a linear-quadratic optimization problem that leads to the solution of the Modified Filter Algebraic Riccati Equation (MFARE). [1418] Adopting this concept to the switched system approach, the goal is to find a minimum dwell time for the switched $\mathcal{H}_{\infty}$ filters. This dwell time constraint then will be used as a restriction for the switching signal that assures that the estimation error will be asymptotically stable.

The above problem can be posed in two different ways. Finding a common Lyapunov function, which leads to solving a group of Linear Matrix Inequalities (LMIs) for a common dwell time solution. This solution, however, might lead to a very conservative solution causing degraded detection performance which is a major disadvantage of the approach. This design restriction can be relaxed by using multiple Lyapunov functions which is the main contribution presented in this article.

The problem of assuring robustness under minimal possible dwell time represents a design trade-off. On the one hand, the filter should be made robust according to the specified $\mathcal{H}_{\infty}$ performance level. On the other hand, the design should provide the minimal possible dwell time between two consecutive switching.

This poses additional requirements for the design of the fault detection filter, requiring the extension of the standard $\mathcal{H}_{\infty}$ filter design problem with the finding of the multiple Lyapunov functions satisfying the dwell time condition.
The paper is organized as follows. In Section 2 the application of the idea of $\mathcal{H}_{\infty}$ optimization to a switched linear system is formulated. In Section 3 the solution method of the determination of the common minimum dwell time is discussed. In Section 4 we demonstrate the applicability of the results to the fault detection filter design for detection of faults in the air path of a diesel engine.

\section{The Concept of Switched $\mathcal{H}_{\infty}$ Fault Detection Filter}

Gain scheduling is a widely used technique for controlling certain classes of nonlinear or linear time-varying systems. This concept for purpose of the solution of robust control problems have been studied extensively in earlier works and useful results are now available. [7, 13, 19, 20, 21] Based on this idea, we pursue a similar solution method for the purpose of application to the robust fault detection filter design problem. According to this method, rather than seeking a single robust linear state estimator and specifying a single $\mathcal{H}_{\infty}$ filter for the entire operating range, one has to design a linear state estimator for each relevant operating point of the system with respective $\mathcal{H}_{\infty}$ constraints. By assigning these filters to specific operating conditions and switching between the corresponding filters as the operating conditions change the design objective can be fulfilled.

\subsection{The Switched Linear System}

Consider the nominal representation of the LTI switched linear system in the form

$$
\begin{aligned}
& \dot{x}(t)=A_{\sigma(t)} x(t)+B_{\sigma(t)} u(t), x(0)=\xi, \\
& y(\mathrm{t})=C_{\sigma(t)} x(t),
\end{aligned}
$$

where for all $t \geq 0, x(t) \in \mathbb{R}^{n}$ is the state vector, $\xi \in \mathbb{R}^{n}$ is the arbitrary fixed initial condition, $u(t) \in \mathbb{R}^{m}$ is the input vector, $y(t) \in \mathbb{R}^{p}$ is the output vector, $\sigma(t):[0, \infty) \rightarrow \Theta$ is the piecewise constant switching function. $A_{\sigma(t)} \in$ $\mathbb{R}^{n x n}, B_{\sigma(t)} \in \mathbb{R}^{n x m}$ and $C_{\sigma(t)} \in \mathbb{R}^{p x n} \quad$ are appropriate constant matrices. Assume, that the pairs $\left(A_{\sigma(t)}, C_{\sigma(t)}\right)$ are observable for all $t \geq 0$. For further consideration let $\Theta=\left\{1, \ldots, n_{e}\right\}$ is the filter set consisting of $n_{e}$ number of filters. The index set $q=1, \ldots, n_{e}$ denotes the sequence number of the switching.

One can extend the system representation in (1) with the concept of perturbed system. [17] The switched linear system subject to disturbance and faults can be represented as follows:

$$
\begin{aligned}
& \dot{x}(t)=A_{\sigma(t)} x(t)+B_{\sigma(t)} u(t)+ \\
& +B_{\kappa \sigma(t)} \kappa(t)+\sum_{i=1}^{k} L_{i \sigma(t)} v_{i}(t), x(0)=\xi, \\
& y(\mathrm{t})=C_{\sigma(t)} x(t),
\end{aligned}
$$

where $B_{\kappa \sigma(t)}=\left[B_{w}, L_{\Delta}\right]$ denotes the worst-case input 
direction and $\kappa(t) \in L_{2}[0, T]$ is the input function for all $t \in \mathbb{R}_{+}$representing the worst-case effects of modelling uncertainties and external disturbances. It is to note, that the (2) does not include parametric uncertainty. [17] The cumulative effect of a number of $k$ faults appearing in known directions $L_{i}$ of the state space is modelled by the additive linear term $\sum L_{i \sigma(t)} v_{i}(t)$ with $L_{i} \in \mathbb{R}^{n x s}$ and $v_{i}(t)$ are the fault signatures and failure modes respectively. $v_{i}(t)$ are arbitrary unknown time functions for $t \geq t_{j i}, 0 \leq t \leq T$, where $t_{j i}$ is the time instant when the $i$-th fault appears and $v_{i}=0$, if $t<t_{j i}$. If $v_{i}(t)=0$, for every $i$, then the plant is assumed fault free. Suppose, moreover, that only one fault appears in the system at a time.

\subsection{The Switched Linear $\mathcal{H}_{\infty}$ Filter}

The state estimator for the system description (2) can be represented by the switched system as follows. Let $z \in \mathbb{R}^{p}$ denote the output signal, then the state estimate can be obtained as

$$
\begin{aligned}
& \dot{\hat{\mathrm{x}}}(t)=\left(A_{\sigma(t)}-K_{\sigma(t)} C_{\sigma(t)}\right) \hat{x}(t)+ \\
& +B_{\sigma(t)} u(t)+K_{\sigma(t)} \mathrm{y}(t), \\
& \hat{\mathrm{y}}(t)=C_{\sigma(t)} \widehat{x}(t), \\
& \mathrm{z}(\mathrm{t})=\mathrm{C}_{z \sigma(t)} \hat{x}(t),
\end{aligned}
$$

where $\hat{x} \in \mathbb{R}^{\mathrm{n}}$ represents the observer state, $\hat{\mathrm{y}} \in \mathbb{R}^{\mathrm{p}}$ represents the output estimate, and $\hat{\mathrm{z}} \in \mathbb{R}^{\mathrm{p}}$ is the weighted output estimate, $K_{\sigma(t)}$ is the observer gain matrix and $C_{z \sigma(t)}$ is the estimation weighting.

The filter error system of (3) is

$$
\begin{aligned}
& \dot{\tilde{\mathrm{x}}}(t)=\left(A_{\sigma(t)}-K_{\sigma(t)} C_{\sigma(t)}\right) \tilde{\mathrm{x}}(t)+ \\
& +B_{\kappa \sigma(t)} w(t)+\sum_{i=1}^{k} L_{i \sigma(t)} v_{i}(t), \\
& \varepsilon(t)=\mathrm{C}_{z \sigma(t)} \tilde{\mathrm{x}}(t),
\end{aligned}
$$

where $\tilde{x}(\mathrm{t})$ and $\varepsilon(t)$ are the state error and weighted output error defined respectively as

$$
\begin{aligned}
& \tilde{x}(t)=x(t)-\widehat{x}(t), \\
& \varepsilon(t)=z(t)-\hat{z}(t) .
\end{aligned}
$$

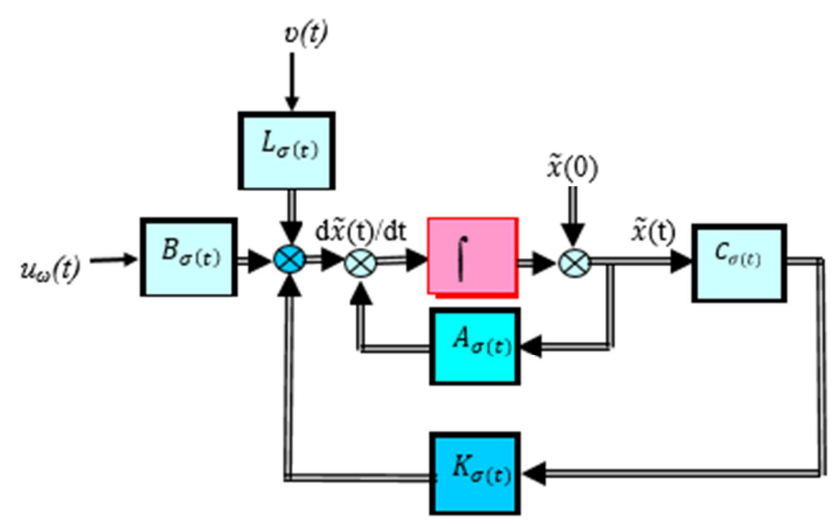

Figure 1. Scheme of the filter estimation error system.
The scheme of the filter estimation error system of the switched state estimator in acc. with (4) is showed in Figure 1.

According to the robust $\mathcal{H}_{\infty}$ filtering problem the quadratic cost function is composed of the weighted output error and worst-case unknown inputs. The extension of this idea to the switched linear system is defined as

$$
\begin{gathered}
J(w, v, \widehat{z}, \sigma)= \\
=\frac{1}{2}\left[\|z-\widehat{z}\|_{2}^{2}-\gamma_{\sigma(t)}^{2}\left(\|w\|_{2}^{2}+\|v\|_{2}^{2}\right)\right],
\end{gathered}
$$

where $\gamma_{\sigma(t)}>0$ is a positive constant. The goal is to find an estimate $\hat{z}$ which minimizes the cost function in (6) under the worst-case input assumption. Technically this means the minimization of the $\mathcal{H}_{\infty}$ norm of the transfer function from the worst-case input to the filter output denoted by $H_{\mathcal{E}_{\kappa}}$.

$$
J(\mathrm{~K}, \kappa, \sigma)=\sup \frac{\left\|z-\widehat{z}_{2}\right\|_{2}}{\|\kappa\|_{2}}=\left\|H_{\varepsilon \kappa}(s)\right\|_{\infty} .
$$

The filter gain $K_{\sigma(t)}$ can be obtained by solving the standard linear-quadratic optimization problem with $\mathrm{H}$ infinity constraint. The goal of the linear-quadratic optimization is to obtain the smallest $L_{2}$-gain of the disturbance input that is guaranteed to be smaller than a positive constant $\gamma_{\min \sigma(t)}$.

The observer Eq. (3) can be rewritten as

$$
\begin{aligned}
& \dot{\hat{\mathrm{x}}}(t)=\left(A_{\sigma(t)}-Y_{\sigma(t)} C_{\sigma(t)}^{T} C_{\sigma(t)}\right) \hat{x}(t)+ \\
& +B_{\sigma(t)} u(t)+Y_{\sigma(t)} C_{\sigma(t)}^{T} \hat{\mathrm{y}}(t), \\
& \hat{\mathrm{z}}(\mathrm{t})=\mathrm{C}_{z \sigma(t)} \hat{x}(t) .
\end{aligned}
$$

From the bounded-real lemma we have $\left\|H_{\varepsilon \kappa}\right\|_{\infty}<$ $\gamma_{\min \sigma(t)}$, if and only if there exists $Y_{\sigma(t)}>0$, such that the MFARE for all $\sigma(t):[0, \infty) \rightarrow \Theta$ can be defined as

$$
\begin{aligned}
& A_{\sigma(t)} Y_{\sigma(t)}+Y_{\sigma(t)} A_{\sigma(t)}^{T}- \\
& -Y_{\sigma(t)}\left(C_{\sigma(t)}^{T} C_{\sigma(t)}-\frac{1}{\gamma_{\sigma(t)}^{2}} C_{z_{\sigma(t)}}^{T} C_{z_{\sigma(t)}}\right) Y_{\sigma(t)}+ \\
& +B_{\kappa_{\sigma(t)}} B_{\kappa_{\sigma(t)}}^{T}=0,
\end{aligned}
$$

where $Y_{\sigma(t)} \in R^{n x n}$ is the positive definite decision variable corresponding to the solution of the respective MFARE.

However, since the asymptotic stability of the state estimation error (4) during the switching has to be ensured, solving the MFARE (9) does not deliver the optimal solution and the $\gamma_{\min \sigma(t)}$ minimal disturbance magnification level as well. This concept has to be extended with considering the minimum dwell time constraint.

A general switching scheme for the problem defined above can be seen in the Figure 2. A number of state observers $\left(1, \ldots, n_{e}\right)$ are applied to the nonlinear process $P$ in parallel. Then, a switching supervisor $S(t)$ governs the switching process at each instant of time based on the input signal $h(t)$ by selecting one out of the $n_{e}$ available observers based 
on the value of the scheduling variable $\sigma(t):[0, \infty)$ generated by $S(t)$. [22] This concept has been successfully applied to switching controller design in the past. $[7,9,10]$

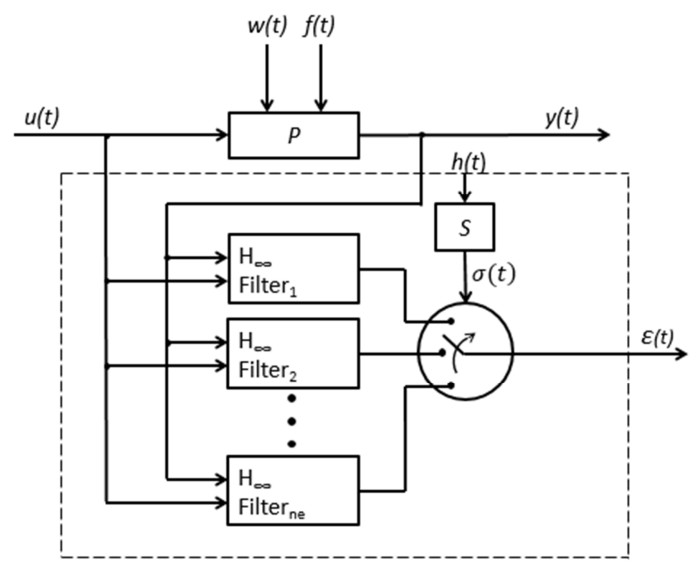

Figure 2. General scheme of the switched filter architecture with using $n_{e}$ observers.

\section{Filter Gain Specification with Dwell Time Constraint}

Ensuring stability of the state estimation error dynamics is the crucial part of the design of the switched filtering scheme characterised in the previous section. It is our basic assumption that the matrices $A_{q}, q \in \Theta$ are Hurwitz, consequently the corresponding systems are asymptotically stable. It follows that the switched stability can be ensured when we switch slowly enough between the subsystems in order to let the transients to dissipate. [9-11]

\subsection{Dwell Time Condition for Switched Filter Stability}

Let us define the total length of the time needed by a particular subsystem to fully falloff in its transient and call it the dwell time $\tau_{\mathrm{D}}>0$. [9, 19, 20,23]

Let $t_{\ell}$ and $t_{\ell+1}$ denote two successive switching times satisfying $t_{\ell+1}-t_{\ell} \geq \tau_{\mathrm{D}}$. Then the selection of the piecewise constant switching function defined as

$$
\sigma(t):[0, \infty) \rightarrow \Theta \text { for all } t\left(t_{\ell}, t_{\ell+1}\right]
$$

ensures, that the equilibrium point $x=0$ of the system in (1) is globally asymptotically stable. Consequently, when designing a switched filtering scheme, we also have to make sure that the time difference between two consecutive switching is not smaller than $\tau_{\mathrm{D}}$, thus the asymptotical stability of the switched linear system is preserved.

The following discussion introduces the problem of stability of switched linear systems for nominal disturbance free cases. [13, 19]

Consider the representation of the continuous-time switched linear system as

$$
x(t)=A_{\sigma(t)} x(t), x(0)=x_{0},
$$

for any $\sigma(t):[0, \infty) \rightarrow \Theta$. As the switching occurs within the finite set of $q \in \Theta=\left\{1, \ldots, n_{e}\right\}$ subsystems, the system description in (10) can be simply represented by the matrices $A_{q}$ as

$$
x(t)=A_{q} x(t), x(0)=x_{0},
$$

for any $q \in \Theta$. Let us assume that the matrices $A_{q}$ are Hurwitz. Consequently, the corresponding subsystems are asymptotically stable for all $q \in \Theta$.

The asymptotic stability of the switched system (11) for any admissible switching signal $\sigma(t)$ is satisfied, when along an arbitrary $x(t)$ trajectory the Lyapunov function and also its derivative satisfy

$$
\begin{aligned}
& V(x(t))=x^{\mathrm{T}}(t) P_{q} x(t)>0, \\
& \dot{V}(x(t))=x^{\mathrm{T}}(t)\left(A_{q}^{\mathrm{T}} P_{q}+P_{q} A_{q}\right) x(t)<0,
\end{aligned}
$$

for any $q \in \Theta, x(t) \neq 0$ and $P_{q} \in R^{n x n}>0$.

For all $t \in\left(t_{\ell}, t_{\ell+1}\right]$, where $t_{\ell+1}=t_{\ell}+T_{\ell},\left(\ell=0, . ., n_{\ell}-\right.$ 1) with $T_{\ell} \geq T_{d}>0$ and $n_{\ell}$ is the number of the switching and at $t=t_{\ell+1}$ the switching jumps to $\sigma(t)=j \in \Theta$.

Assuming that for some $T_{d}>0$ there exists a collection of positive definite matrices $\left\{P_{1}, \ldots, P_{n e}\right\}$ of compatible dimensions such that the LMIs

$$
\begin{aligned}
& A_{q}{ }^{T} P_{q}+P_{q} A_{q}<0, \forall q \in \Theta, \\
& e^{A_{q}{ }^{T} T_{d}} P_{j} e^{A_{q} T_{d}}-P_{q}<0, \forall q \neq j \in \Theta,
\end{aligned}
$$

hold, then the time switching control of $\sigma(t):[0, \infty) \rightarrow \Theta$ makes the equlibrium solution $x=0$ globally asymptotically stable.

It is seen from (13) that for all $t \in\left(t_{\ell}, t_{\ell+1}\right]$ the time derivative of the Lyapunov function (12) along an arbitrary trajectory of (11) is satisfied. That ensures, that there exist some $\alpha>0$ and $\beta>0$ scalars for wich for all $t \in\left(t_{\ell}, t_{\ell+1}\right]$ is satisfied, that

$$
\|x(t)\|^{2} \leq \beta e^{-\alpha\left(t-t_{\ell}\right)} V\left(x\left(t_{\ell}\right)\right) .
$$

For two consecutive switching $t_{\ell}, t_{\ell+1}$ the Lyapunov function (10) becomes

$$
\begin{aligned}
V\left(x\left(t_{\ell+1}\right)\right) & =x\left(t_{\ell+1}\right)^{\mathrm{T}} P_{j} x\left(t_{\ell+1}\right)= \\
& =x\left(t_{\ell}\right)^{\mathrm{T}} e^{A_{q}{ }^{T} T_{\ell}} P_{j} e^{A_{q} T_{\ell}} x\left(t_{\ell}\right) \\
& <x\left(t_{\ell}\right)^{\mathrm{T}} e^{A_{q}{ }^{T}\left(T_{\ell}-T_{d}\right)} P_{q} e^{A_{q}\left(T_{\ell}-T_{d}\right)} x\left(t_{\ell}\right) \\
& <x\left(t_{\ell}\right)^{\mathrm{T}} P_{q} x\left(t_{\ell}\right)<V\left(x\left(t_{\ell}\right)\right)
\end{aligned}
$$

where the second inequality holds due to the fact that

$$
e^{A_{q} T_{d}} P_{q} e^{A_{q} T_{d}} \leq P_{q}
$$

is true for every $\tau=T_{\ell}-T_{d}>0$. Consequently, there exists $\mu \in(0,1)$ such that 


$$
V\left(x\left(t_{l}\right)\right) \leq \mu^{l} V(x(0)), \forall l \in \Theta,
$$

which, together with (14) implies that the equilibrium solution $x=0$ is globally asymptotically stable. [19] This means that the sequence of Lyapunov functions are positive and decreasing.

Based on this, the filter synthesis technique proposed in the following part is originated in the results from the research of Geromel Jose C. and Colaneri Patrizio in 2008. [19] By adopting their results formulated in the robust nonlinear control problem to the framework of $\mathcal{H}_{\infty}$ detection filter design by dualization. However, in contrast with this, where a single worst-case $\gamma$ for all controllers together with the related dwell time is calculated, in our present paper we solve the dwell time optimization problem for each specific $\mathcal{H}_{\infty}$ performance level. This approach, as it is expected, will result in a less conservative filtering scheme with improved detection performance.

\section{2. $\mathcal{H}_{\infty}$ Filter Synthesis Involving Dwell Time Constraint}

In the switched $\mathcal{H}_{\infty}$ detection filter design problem our goal is to find a common minimum dwell time, that assures the estimation error will be asymptotically stable for all specified $\mathcal{H}_{\infty}$ level calculated separately for each filter. It was shown that following this procedure the robust stability of the estimation error system (4) can be preserved under the worstcase disturbance.

The direct solution of this problem associated with the Hamilton-Jacobi-Bellmann equation for any given dwell time would be extremely difficult due to the algebraic structure of the set $\Theta$. [19] A more realistic interpretation of the problem can be given by using a two-step design procedure that can be formulated as follows: (i) solve the MAFARE and calculate the $\mathcal{H}_{\infty}$ constrained filter gain for each particular subsystem, then (ii) determine the subjected common minimum possible dwell time for all $q \in \Theta$. This takes the determination of $\mathrm{T}_{\mathrm{dmin}}\left(\gamma_{d}\right)$, for specified $\gamma_{d q} \geq \gamma_{\text {minq }}$ for all $q \in \Theta$ such that $\sigma(t):[0, \infty) \rightarrow \Theta$ and $\mathrm{t}_{\ell+1}-\mathrm{t}_{\ell} \geq$ $\mathrm{T}_{\mathrm{dmin}}$ hold.

The MFARE for each $q \in \Theta$ is defined as

$$
\begin{aligned}
& A_{q} Y_{q}+Y_{q} A_{q}^{T}- \\
& -Y_{q}\left(C_{q}^{T} C_{q}-\frac{1}{\gamma_{q}^{2}} C_{z q}^{T} C_{z q}\right) Y_{q}+ \\
& +B_{\kappa q} B_{\kappa q}^{T}=0,
\end{aligned}
$$

which can be factorized by using the transformations as

$$
\begin{aligned}
& A_{q} Y_{q}+Y_{q} A_{q}^{T}-Y_{q}\left[C_{z q}^{T} C_{q}^{T}\right] \\
& {\left[\begin{array}{cc}
-\frac{1}{\gamma_{q}^{2}} & 0 \\
0 & I
\end{array}\right]\left[\begin{array}{c}
C_{z q} \\
C_{q}
\end{array}\right] Y_{q}+B_{\kappa q} B_{\kappa q}^{T}=0}
\end{aligned}
$$

$$
\begin{aligned}
& W_{q}=Y_{q}\left[C_{z q}^{T} C_{q}^{T}\right]\left[\begin{array}{cc}
-\frac{1}{\gamma_{q}^{2}} I & 0 \\
0 & I
\end{array}\right], \\
& \left(A_{q}-W_{q}\left[\begin{array}{c}
C_{z q} \\
C_{q}
\end{array}\right]\right) Y_{q}+Y_{q}\left(A_{q}-W_{q}\left[\begin{array}{c}
C_{z q} \\
C_{q}
\end{array}\right]\right)^{T}+ \\
& +Y_{q}\left[\begin{array}{c}
C_{z q} \\
C_{q}
\end{array}\right]^{T} W_{q}^{T}+B_{\kappa q} B_{\kappa q}^{T}=0, \\
& \left(A_{q}-W_{q}\left[\begin{array}{c}
C_{z q} \\
C_{q}
\end{array}\right]\right) Y_{q}+Y_{q}\left(A_{q}-W_{q}\left[\begin{array}{c}
C_{z q} \\
C_{q}
\end{array}\right]\right)^{T}+ \\
& +W_{q}\left[\begin{array}{cc}
-\frac{1}{\gamma_{q}^{2}} I & 0 \\
0 & I
\end{array}\right]^{-1} W_{q}^{T}+B_{\kappa q} B_{\kappa q}^{T}=0, \\
& \left(A_{q}-W_{q}\left[\begin{array}{c}
C_{z q} \\
C_{q}
\end{array}\right]\right) Y_{q}+ \\
& +Y_{q}\left(A_{q}-W_{q}\left[\begin{array}{c}
C_{z q} \\
C_{q}
\end{array}\right]\right)^{T}+ \\
& +\left(W_{q}\left[\begin{array}{ll}
0 & 0 \\
0 & I
\end{array}\right] W_{q}^{T}+B_{\kappa q} B_{\kappa q}^{T}\right)- \\
& -\gamma_{q}^{2} W_{q}\left[\begin{array}{ll}
I & 0 \\
0 & 0
\end{array}\right] W_{q}^{T}=0 .
\end{aligned}
$$

Introducing the matrix notation

$$
\begin{gathered}
H_{q}=\left(A_{q}-W_{q}\left[\begin{array}{c}
C_{z q} \\
C_{q}
\end{array}\right]\right), \\
Q_{q}=\left(W_{q}\left[\begin{array}{ll}
0 & 0 \\
0 & I
\end{array}\right] W_{q}^{T}+B_{\kappa q} B_{\kappa q}^{T}\right)- \\
-\gamma_{q}^{2} W_{q}\left[\begin{array}{ll}
I & 0 \\
0 & 0
\end{array}\right] W_{q}^{T} .
\end{gathered}
$$

the final form of the factorization of (18) reduces to the Riccati equation

$$
H_{q} Y_{q}+Y_{q} H_{q}^{T}+Q_{q}=0, \forall q \in \Theta .
$$

Note that the optimal gain $W_{q}$ is determined by the unique stabilizing solution of the MFARE (18) such that the matrix $H_{q}$ is Hurwitz for each $q \in \Theta$. The Riccati equation (26) admits a positive definite solution since it was created by means of the factorization of the MFARE (18).

Assume that for any $\sigma(t):[0, \infty) \rightarrow \Theta$ and for all $t \in$ $\left(t_{\ell}, t_{\ell+1}\right]$, where $t_{\ell+1}=t_{\ell}+T_{\ell}$ with $T_{\ell} \geq T_{d}>0$ and at $t=t_{\ell+1}$ the switching jumps to $\sigma(t)=j \in \Theta$, where the corresponding solution of the Lyapunov function along a 
trajectory of the switched filter error system is expressed by

$$
\begin{aligned}
\mathrm{V}\left(\tilde{\mathrm{x}}\left(t_{\ell}\right)\right) & =\tilde{\mathrm{x}}\left(t_{\ell+1}\right)^{\mathrm{T}} Z_{j} \tilde{\mathrm{x}}\left(t_{\ell+1}\right)= \\
& =\tilde{\mathrm{x}}\left(t_{\ell}\right)^{\mathrm{T}} e^{{ }^{H_{q}} T^{T}{ }} Z_{j} e^{H_{q} T_{\ell}} \tilde{\mathrm{x}}\left(t_{\ell}\right) .
\end{aligned}
$$

The above already mentioned $\mathcal{H}_{\infty}$ state feedback control problem can be associated to the corresponding $\mathcal{H}_{\infty}$ filtering problem by duality. [19] Based on (26) and the Lyapunov function (27) formulated along a trajectory of the state estimation error system in (4) one can derive the LMI which can be used to obtain a common minimum dwell time for all specified $\mathcal{H}_{\infty}$ level in the following way.

Assume that for a given $T_{d}$ there exists a collection of positive definite matrices $\left\{Z_{1}, \ldots, Z_{n e}\right\}$ of compatible dimensions such that the LMIs

$$
\begin{aligned}
& H_{q} Z_{q}+Z_{q} H_{q}{ }^{T}+Q_{q}<0, \forall q \in \Theta \\
& e^{H_{q} T_{d}} Z_{j} e^{H_{q} T_{d}}-Z_{q}+Y_{q}<0, \forall q \neq j \in \Theta
\end{aligned}
$$

hold. Then under the worst-case input assumption (2) for any switching signal $\sigma(t):[0, \infty) \rightarrow \Theta$ satisfying the condition $T_{d}$ $=t_{\ell+1}-t_{\ell} \geq T_{d m i n}$. Then, the equilibrium solution of the state estimation error system (4) is globally asymptotically stable. Additionally for all $q \in \Theta$ filters is ensured a $\gamma_{d q} \geq$ $\gamma_{\text {minq }}$ minimal disturbance magnification level.

Because of the matrix $Q_{q}$ in (26) depends on the value of $\gamma_{\text {minq }}$, it is not positive definite for any $q \in \Theta$. Note that for the feasibility of the LMIs in (28) as well as for existing $T_{d}$ the satisfaction of the condition $Q_{q} \geq 0$ is necessary. In other cases, however, $\gamma_{\mathrm{q}}>\gamma_{\text {minq }}$ can always be chosen such that $Q_{q} \geq 0$ holds.

To sum up, solving the LMI (28) the common minimum dwell time $T_{d \min }$ for each $\mathcal{H}_{\infty}$ performance level can be calculated by searching the minimum of $T_{d}$.

This calculation can be carried out by performing the followings procedure:

1. For each $q \in \Theta$ one has to solve the MFARE (18) for $Y_{q}$ and $\gamma_{q \min }$, then calculate $W_{q}$ by (20).

2. From the formulas in (24) and (25) the matrices $H_{q}$ and $Q_{q}$ can be calculated, in a respective way.

3. If the condition $Q_{q} \geq 0$ doesn't hold, then choose $\gamma_{\mathrm{dq}}$ $>\gamma_{\text {minq }}$ such that $Q_{q} \geq 0$ holds. Then recalculate $Y_{q}$ and $H_{q}$, accordingly

4. Formulate the LMIs in (28) as a multivariable feasibility optimization problem for each $q \in \Theta$.

5. Initialize the $T_{d}>0$, arbitrary.

6. Calculate the matrix exponent $e^{H_{q} T_{d}}$ for this $T_{d}$.

7. Solve the LMIs in (28) iteratively by successive reduction of $T_{d}$ until the feasibility constraint for $Z_{q}$ satisfies. The minimal value of $T_{d}=T_{d \min }$ is obtained as the common minimum dwell time.

By using the solution of (18) the filter gain matrix for any $q \in \Theta$ can be obtained as

$$
K_{q}=Y_{q} \mathrm{C}_{q}^{T}
$$

With the use of $\gamma_{\text {qmin }}$ the detection threshold of the filter for any $q \in \Theta$ is given

$$
\tau_{q}\left(C_{z}\right)=\gamma_{q \min }\|\kappa\|_{2} .
$$

The transfer function from the unknown input to the filter residual for a given filter gain $K_{q}$ can be written as

$$
G_{q e x}(\mathrm{~s})=C_{Z}\left(\mathrm{sI}-A_{q}+K_{q} C_{q}\right)^{-1} B_{q \kappa} .
$$

Analogously, the transfer function from the fault to the filter residual for the given filter gain $K_{q}$ is obtained as

$$
G_{q \varepsilon v}(\mathrm{~s})=C_{Z}\left(\mathrm{sI}-A_{q}+K_{q} C_{q}\right)^{-1} L_{q i} .
$$

The upper bound for the $\mathcal{H}_{\infty}$ attenuation level of the switched filter system is, therefore

$$
\gamma_{u b}>\max _{q \in \Theta} G_{q \varepsilon \kappa}(\mathrm{s}) .
$$

Then, the guaranteed filter sensitivity can be defined as

$$
S_{l b}=\frac{\left\|G_{q \varepsilon v}(s)\right\|_{\infty}}{\max _{q \in \Theta}\left\|G_{q \varepsilon \kappa}(s)\right\|_{\infty}} .
$$

\subsection{Solution of the Constrained MFARE}

It was shown in the previous sections that the problem of finding a common minimum dwell time for every specified $\mathcal{H}_{\infty}$ performance level can be solved in the framework of LMI representations. In this section the solution process is detailed as follows.

At first, we have to solve the corresponding MFARE in (18) for each particular subsystem $q \in \Theta$. It is explained how the MFARE in the LMI framework can be formulated. [12]

We can get the LMI for the q-th subsystem by letting $R_{q}=Y_{q}^{-1}$ and applying the Schur lemma. [18]

$$
\left[\begin{array}{ccc}
R_{q} A_{q}+A_{q}^{T} R_{q}-C_{q}^{T} C_{q} & C_{z q}^{T} & R_{q} B_{* q} \\
C_{z q} & -\gamma_{q}^{2} I & 0 \\
B_{\kappa q}^{T} R_{q} & 0 & -I
\end{array}\right]<0,
$$

which has a solution $R_{q}=R_{q}^{T}, R_{q}>0 \in \mathbb{R}^{n x m}$ for $\gamma_{q}>0$.

Consequently, the MFARE minimizing $\gamma_{q}$ with respect to $R_{q}>0$ subject to (35) can be sought in the following optimization problem:

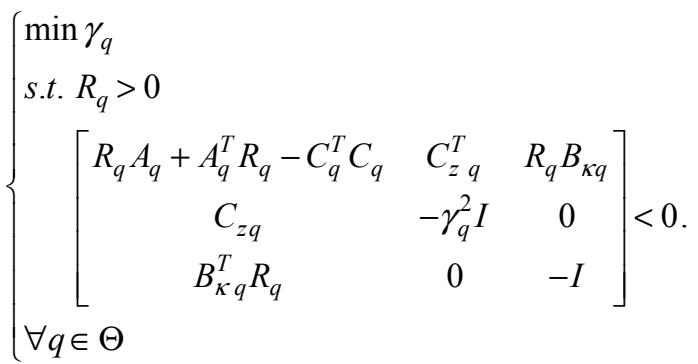


The LMI in (36) is formulated as a linear objective minimization problem that can be solved by using the mincx function of the LMI Control Toolbox in MATLAB. [23, 25] The corresponding MATLAB code for the solution of the LMI formulation (36) is described by Horváth Zsolt and Edelmayer András. [18]

In the following we pursue finding the mimimum dwell time for each subsystem $q \in \Theta$, that can be done for fixed $\gamma_{q}$ obtained from the solution of the LMIs in (36). This can be posed as the following time dependent multivariable optimization problem:

$$
\begin{cases}\min & T_{d} \\ \text { s.t. } & Z_{q}>0 \\ & Z_{j}>0 \\ & H_{q} Z_{q}+Z_{q} H_{q}{ }^{T}+Q_{q}<0 \\ & e^{H_{q} T_{d}} Z_{j} e^{H_{q}{ }^{T} T_{d}}-Z_{q}+X_{q}<0 . \\ \forall q & \neq j \in \Theta\end{cases}
$$

Since the matrix-exponential, which includes the design variable $T_{d}$ is nonlinear, the LMI in (37) cannot be treated as a simple scalar value minimization and casted as a linear objective minimization problem, and the value $T_{d}$ is immediately obtained. [25, 23, 27]

In order to overcome this difficulty, we implemented an algorithm called $T_{d}$-iteration, which is based on interval halving. The algorithm decreases the value of $T_{d}$ until the constraints of the LMIs (28) are no longer feasible, consequently any of $Z_{q}, q \in \Theta$, have no longer a positive definite solution. The $T_{d \min }$ which is so reached, is within the limits given by an arbitrarily small tolerance $\varepsilon^{\prime}>0$ and is the common minimum dwell time for each specified $\mathcal{H}_{\infty}$ performance level, thus it holds, that $T_{d \min } \leq \tau_{D}$. For the the feasibility solution of the LMIs the combination of the interval halving method with the standard LMI solver seems computationally efficient. The complete algorithm for $T_{d}$ iteration was earlier presented. [28-29]

\section{Switched $\mathcal{H}_{\infty}$ Filter Design for Fault Detection in the Air Path of a Diesel Engine}

In the following part the above characterised switched filter design is applied to the detection of faults in the air path of diesel engines.

\subsection{Model Approach}

A simplified nonlinear model of the air path of diesel engines was first proposed for purpose of robust control. [30] In our earlier investigations we adopted the linearized version of this model at a chosen operating point to get an LTI formulation of the filter synthesis. [31] The corresponding linear $\mathrm{H}$-infinity detection filter solution was published that showed promising single point performance. $[14,18]$
Following the idea presented in this article a switched linear system model was developed with choosing 64 operating points along the whole trajectory. This corresponds to the most typical low and medium speed load points of the engine covering the New European Drive Cycle (NEDC) specification. [30] The inputs of the switched representation (2) are the actuation signals of the Exhaust Gas Recirculation Valve (EGR-Actuator) and Variable Geometry Turbocharger (VGTActuator). For gross simplification, we considered fuelling as a constant input of the air path. The disturbance was modelled as a fluctuating change of the engine speed, which is normally caused by the variable load during the engine's operation.

For simplicity we linearized the system in 7 operating points only, letting to derive 7 stable LTI-systems from the nonlinear representation. [22] The set of 7 LTI systems are then used to design the $\mathcal{H}_{\infty}$ detection filters.

According to the design method presented in Section 3 we have to solve the MFARE (18) as linear minimization problem in (36) for each subjected subsystem $q \in \Theta$ in LMI formulation. The solution method was presented in our earlier investigation with using the LMI-Toolbox in MATLAB. [18]

\subsection{Filter Design}

Based on the above $\gamma_{\text {qmin }}$ and $Y_{q}$ are obtained, then the matrices $H_{q}$ and $Q_{q}$ calculated in accordance with (24) (25). Note that in this case the condition $Q_{q} \geq 0$ was not satisfied, therefore, $\gamma_{\mathrm{dq}}>\gamma_{\text {minq }}$ were chosen such, that $Q_{q} \geq 0$ holds, iteratively. Then $Y_{q}$ and $H_{q}$ are recalculated, accordingly.

In order to find the common minimum dwell time, we must solve the feasibility problem in (37) for each subsystem $\mathrm{q} \in \Theta$ using matrices $Y_{q}, H_{q}$ and $Q_{q}$. This can be performed by using the algorithm $T_{d}$-iteration. [28-29] The algorithm reduces the value of $T_{d}$ until the constraints of the LMIs in (28) are no longer feasible, that means for any $Z_{q}, q \in$ $\Theta$ have no longer positive definite solutions. The $T_{d \min }$ which is so reached can be interpreted within the limits given by an arbitrarily small tolerance $\varepsilon>0$.

The common minimum dwell time is represented by $T_{d m i n}$. This means, that between the switches of the filters a bigger waiting time than $T_{d \min }$ is to be applied to allow the filter estimation error system transients to dissipate.

\subsection{Simulation Results}

The individual parameters of the results provided by the $T_{d}$-iteration algorithm implemented for above mentioned 7 linear $\mathcal{H}_{\infty}$ filters are summarized in Table 1 . For the minimum dwell time the value $T_{d \min }=0.106 \mathrm{~s}$ was obtained. In Table 1 the originally specified $\mathcal{H}_{\infty}$-level for each single filter $\gamma_{\text {minq }}$ was increased to $\gamma_{\mathrm{dq}}>\gamma_{\text {minq }}$ such, that the feasibility condition $Q_{q} \geq 0$ holds. The feasible solutions for $Z_{q}$ and the corresponding $Y_{q}$ and $Q_{q}$ matrices are also shown. Finally, the filter gains $K_{q}$ for each subsystem based on the representation (29) are calculated.

In order to show that the designed filter works properly the following computer simulation was compiled and performed. In the system model, bias faults in the VGT-Actuator and 
also in the EGR-Actuator signals were modelled, in which the amplitude of the signals at $2.5 \mathrm{sec}$ was increased up to $30 \%$ of the nominal value, step-wise. This fault enters the system in the same direction of the state space as the input does, which can be represented by an additive term. Detection problem of this kind for linear systems was already discussed in the past. $[14,18]$ A disturbance was modelled as a fluctuation of the engine speed by $10 \%$.

Though the residuals, as shown in Figure 3-4, according to the different filter gains are scattered, the fault signatures can still be safely reproduced.

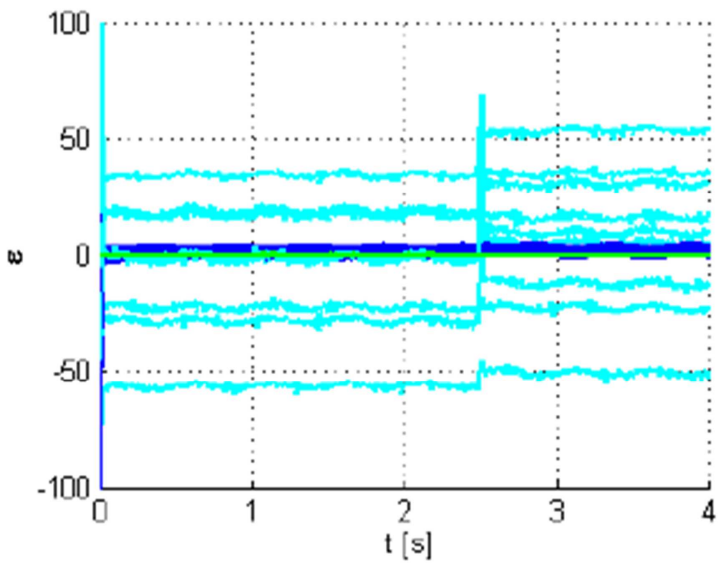

Figure 3. VGT-Actuator bias fault residuals occurring at $t=2.5 \mathrm{sec}$ in the presence of engine speed disturbance. The filter is switched between the 7 operating points in the engine entire operating range. Residuals: $\boldsymbol{\varepsilon}_{\mathbf{1}}$ (blue line), $\boldsymbol{\varepsilon}_{2}$ (cyan line), $\boldsymbol{\varepsilon}_{3}$ (green line).

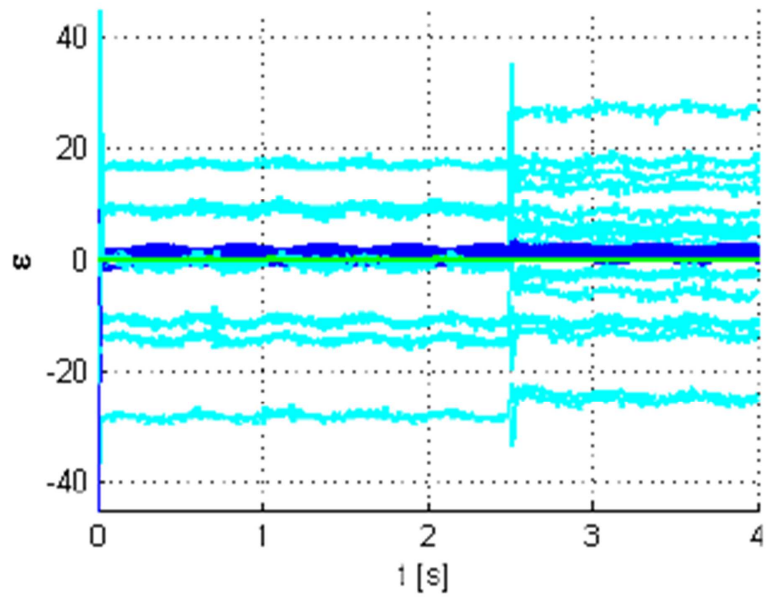

Figure 4. EGR-Actuator bias fault residuals occurring at $t=2.5 \mathrm{sec}$ in the presence of engine speed disturbance. The filter is switched between the 7 operating points in the engine entire operating range. Residuals: $\varepsilon_{1}$ (blue line), $\varepsilon_{2}$ (cyan line), $\varepsilon_{3}$ (green line).

In order to verify filter robustness, the transfer functions calculated from the disturbance and also from the faults to the filter residuals for the corresponding filter gains $K_{q}$ are shown in Figure 5. It can be seen that a proper separation at about $50 \mathrm{~dB}$ can be guaranteed between the disturbance effect and the modelled faults. It can be concluded that this sensitivity is normally satisfactory to detect both faults under the worst-case disturbance effect.

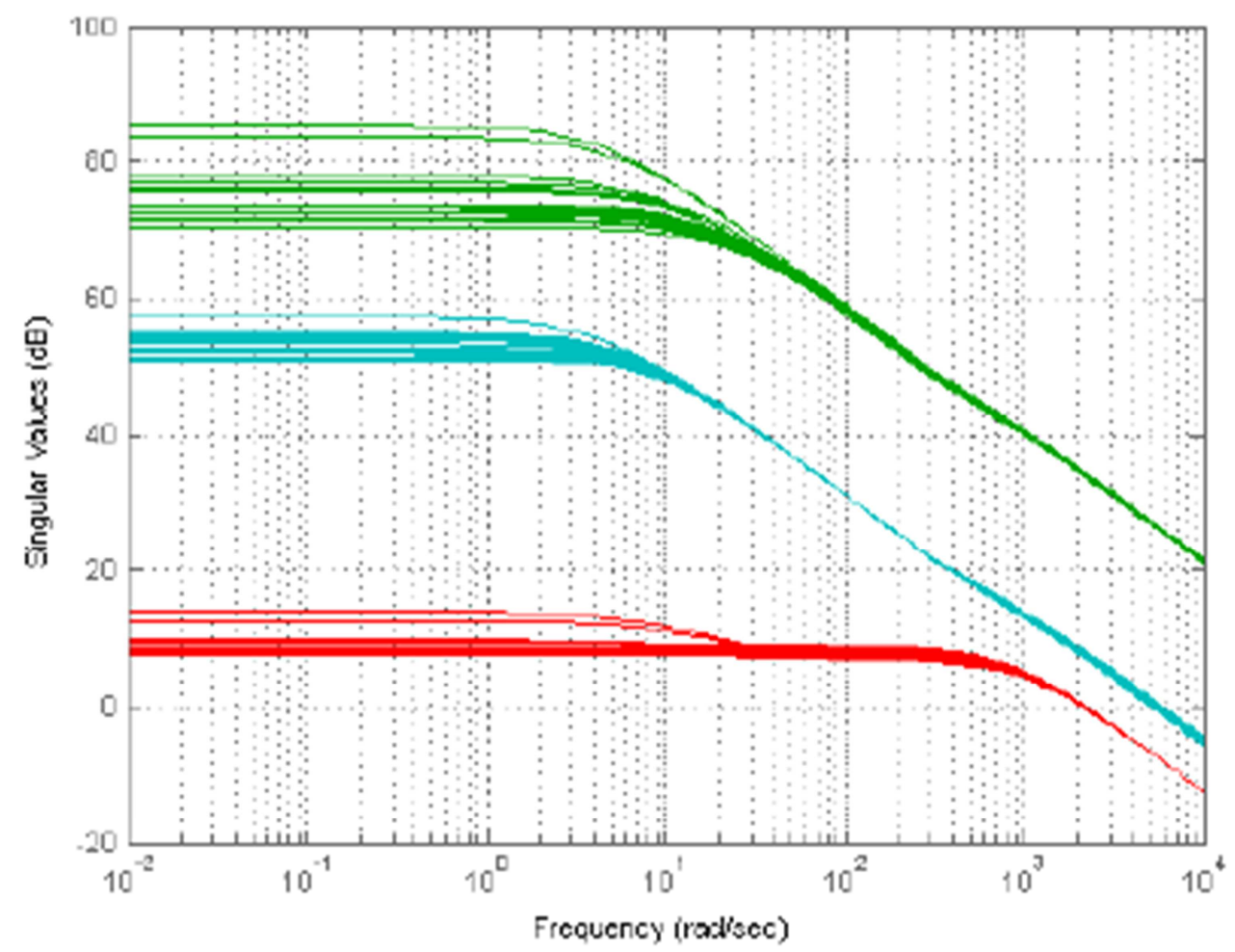

Figure 5. The magnitude (maximal singular values) of the transfer functions: $G_{q \varepsilon \kappa}$ (red line), $G_{q \varepsilon f e g r}\left(\right.$ green line), $G_{q \varepsilon f v g t}(c y a n$ line). 
Table 1. Results provided by the $T_{d}$-iteration algorithm implemented for the 7 linear $\mathcal{H}_{\infty}$ filters.

\begin{tabular}{|c|c|c|c|c|c|c|c|c|c|c|c|}
\hline$q$ & $\gamma_{q \min }$ & $\gamma_{d q}$ & $\mathbf{Q}_{q}$ & & & $\mathbf{Y}_{q}$ & & & $\mathbf{z}_{q}$ & & \\
\hline \multirow{5}{*}{1} & \multirow{4}{*}{4.9224} & \multirow{4}{*}{8.0640} & $10^{5} *$ & & & & & & $10^{4} *$ & & \\
\hline & & & 0.1810 & -0.2182 & -0.0002 & 81.6979 & -17.0197 & -0.1127 & 1.4041 & 0.1807 & 1.6580 \\
\hline & & & -0.2182 & 2.7837 & 0.0006 & -17.0197 & 244.1857 & 0.2370 & 0.1807 & 0.1792 & 0.2614 \\
\hline & & & -0.0002 & 0.0006 & 0.0000 & -0.1127 & 0.2370 & 0.0103 & 1.6580 & 0.2614 & 5.8559 \\
\hline & \multirow{4}{*}{4.8757} & \multirow{5}{*}{8.7323} & $10^{5} *$ & & & & & & $10^{4} *$ & & \\
\hline \multirow{3}{*}{2} & & & 0.1209 & -0.2449 & -0.0002 & 61.5844 & -18.0647 & -0.1088 & 1.8447 & 0.2959 & 2.0206 \\
\hline & & & -0.2449 & 3.0927 & 0.0006 & -18.0647 & 249.8336 & 0.2452 & 0.2959 & 0.1944 & 0.3692 \\
\hline & & & -0.0002 & 0.0006 & 0.0000 & -0.1088 & 0.2452 & 0.0120 & 2.0206 & 0.3692 & 5.8282 \\
\hline \multirow{4}{*}{3} & \multirow{4}{*}{4.8217} & & $10^{5} *$ & & & & & & $10^{4} *$ & & \\
\hline & & \multirow{3}{*}{9.0668} & 0.0854 & -0.2658 & -0.0001 & 48.0245 & -19.4905 & -0.1127 & 2.4749 & 0.4923 & 2.5544 \\
\hline & & & -0.2658 & 3.1959 & 0.0008 & -19.4905 & 250.7915 & 0.2733 & 0.4923 & 0.2441 & 0.5643 \\
\hline & & & -0.0001 & 0.0008 & 0.0000 & -0.1127 & 0.2733 & 0.0142 & 2.5544 & 0.5643 & 5.9937 \\
\hline \multirow{4}{*}{4} & \multirow{4}{*}{4.5688} & \multirow{4}{*}{8.9252} & $10^{5} *$ & & & & & & $10^{4} *$ & & \\
\hline & & & 0.0428 & -0.3092 & -0.0001 & 24.1243 & -21.7061 & -0.1440 & 6.8097 & 2.1976 & 5.0418 \\
\hline & & & -0.3092 & 3.4441 & 0.0006 & -21.7061 & 246.8474 & 0.2150 & 2.1976 & 0.8580 & 1.6961 \\
\hline & & & -0.0001 & 0.0006 & 0.0000 & -0.1440 & 0.2150 & 0.0118 & 5.0418 & 1.6961 & 6.1133 \\
\hline \multirow{4}{*}{5} & \multirow{4}{*}{4.4186} & \multirow{4}{*}{9.3250} & $10^{5} *$ & & & & & & $10^{4} *$ & & \\
\hline & & & 0.0428 & -0.3717 & -0.0001 & 18.4410 & -23.5689 & -0.1205 & 9.8455 & 3.6942 & 6.3182 \\
\hline & & & -0.3717 & 4.0587 & 0.0005 & -23.5689 & 260.8637 & 0.1745 & 3.6942 & 1.5341 & 2.4347 \\
\hline & & & $\begin{array}{c}-0.0001 \\
10^{5} *\end{array}$ & 0.0005 & 0.0000 & -0.1205 & 0.1745 & 0.0088 & $\begin{array}{l}6.3182 \\
10^{5} *\end{array}$ & 2.4347 & 6.0289 \\
\hline \multirow{3}{*}{6} & \multirow{3}{*}{4.1948} & \multirow{3}{*}{10.3314} & 0.0385 & -0.3596 & -0.0001 & 13.2562 & -21.7716 & -0.1215 & 1.4072 & 0.6114 & 0.7877 \\
\hline & & & -0.3596 & 3.8118 & 0.0004 & -21.7716 & 231.8670 & 0.1571 & 0.6114 & 0.2806 & 0.3482 \\
\hline & & & $\begin{array}{l}-0.0001 \\
10^{5} *\end{array}$ & 0.0004 & 0.0000 & -0.1215 & 0.1571 & 0.0093 & $\begin{array}{l}0.7877 \\
10^{5} *\end{array}$ & 0.3482 & 0.6131 \\
\hline \multirow{3}{*}{7} & \multirow{3}{*}{3.7459} & \multirow{3}{*}{11.4722} & 0.0422 & -0.4162 & -0.0000 & 8.0359 & -21.3598 & -0.1014 & 2.5028 & 1.3657 & 1.0974 \\
\hline & & & -0.4162 & 4.2496 & 0.0002 & -21.3598 & 217.5342 & 0.1136 & 1.3657 & 0.7613 & 0.6037 \\
\hline & & & -0.0000 & 0.0002 & 0.0000 & -0.1014 & 0.1136 & 0.0073 & 1.0974 & 0.6037 & 0.6259 \\
\hline
\end{tabular}

\section{Conclusions}

This paper deals with the application of $\mathcal{H}_{\infty}$ detection filters to fault detection in nonlinear systems. The idea is that the nonlinear dynamics in specific meaningful points of the operation is approximated by means of a matched array of linear systems and a linear $\mathcal{H}_{\infty}$ filter are designed for each particular subsystem. Then, a switching scheme is applied to carefully choose the most suitable filter regarding the operational characteristics of the plant in real time. Stability of the switching process is guaranteed by keeping the switching time between two consecutive switching large enough to ensure a proper falloff of filter transients. The goal is to find a common minimum dwell time that can be considered as the worst-case minimal waiting time for the filter transitions to eliminate between consecutive switching. This dwell time can be considered as a restriction for the switching signal that assures that the state estimation error will be asymptotically stable for each specified $\mathcal{H}_{\infty}$ performance level calculated separately for each particular filter.

The solution method presented in this paper is based on the dualized results from the research of Geromel Jose C. and Colaneri Patrizio in 2008. [19] In contrast with this solution method, in our approach the dwell time optimization problem is solved according to each specific $\mathcal{H}_{\infty}$ performance level, i.e., to each specific $\gamma_{q}$ based on the multiple Lyapunov function approach. This approach results in less conservative filtering with improved detection performance. The results can be considered as the extension of the standard linear $\mathcal{H}_{\infty}$ fault detection filtering problem to nonlinear cases.

The simulation results indicate the applicability of the proposed filtering approach to fault detection in nonlinear systems with satisfying sensitivity. Further studies are required to investigate the conditions of real industrial applications.

Though the waiting time between two consecutive switching obtained in our recent simulation study (i.e., 0.16 $s e c$ ) could be tolerated in many slow industrial processes, it may provide a significant limitation for applications in plants with fast dynamics, such as in combustion engine applications. It can be expected that this limitation can be relieved by using a higher resolution filter lattice, i.e., by increasing the number of operating points in the engine's linearized operation range and working with a richer set of filters in the filter lattice, correspondingly. Another possibility is the application of an optimized switching strategy according to which the neighboring filters are considered only for selection instead of relying on a fully connected filter network.

\section{References}

[1] Boulkroune Boulaid, Djemili Issam, Aitouche Abdel, and Cocquempot Vincent. Robust nonlinear observer design for actuator fault detection in diesel engines. International Journal of Applied Mathematics and Computer Science. Vol. 23, No. 3, 2013, pp. 557-569. 
[2] Sangha, M. S, Yu, D. L., and Gomm, J. B. Sensor fault detection, isolation, accommodation and unknown fault detection in automotive engine using AI. International Journal of Engineering, Science and Technology. Vol.4, No.3, 2012, pp. 53-65.

[3] Guermouche Mohamed, Benkaci Mourad, Hoblos Ghaleb and Langlois Nicolas. Sensor fault detection and isolation in diesel air path using fuzzy-ARTMAP neural network. IEEE 10th International Conference on Networking, Sensing and Control (ICNSC). Evry, France, 2013, pp.728-733.

[4] Krishnaswami Venkat, Chun-L G., and Rizzoni Giorgio. Fault Detection in IC Engines using Nonlinear Parity Equations. Proceedings of the American Control Conference. Baltimore, Maryland, 1994, pp. 2001-2005.

[5] Vanek Bálint, Szabó Zoltán, Edelmayer András, Bokor József. Geometric LPV fault detection filter design for commercial aircrafts. AIAA Guidance, Navigation and Control Conference. Portland, OR, United States, 2011.

[6] Edelmayer András, Bokor József, Szabó Zoltán. A geometric view on inversion-based detection filter design in nonlinear systems. In Proceedings of the fifth IFAC symposium on fault detection, supervision and safety of technical processes. SAFEPROCESS, Washington, 2003, pp. 783-788.

[7] Paxman Jonathan. Switching Controllers: Realization, Initialization and Stability. Ph.D Dissertation. University of Cambridge, 2003.

[8] DeCarlo Raymond A., Branicky Michael, Pettersson Stefan and Lennartson Bengt. Perspectives and Results On the Stability and Stabilizability of Hybrid Systems. Proc. IEEE. Vol. 88, 2000, pp. 1069-1082.

[9] Liberzon Daniel, A. Stephen Morse. Basic Problems in Stability and Design of Switched Systems. IEEE Contr. Syst. 1999, pp. 59-70.

[10] João Pedro Hespanha, A. Stephen Morse. Stability of switched systems with average dwell-time. Proc. 38th Conf. Decision and Contr. 1999, pp. 2655-2660.

[11] Alessandri Angelo, Coletta Paolo. Switching observers for continuous-time and discrete-time linear systems. In Proceedings of the American Control Conference Arlington. 2001.

[12] Johansson Mikael. Piecewise Linear Control Systems: A computational Approach. Springer Verlag. Heidelberg, 2003.

[13] Colaneri Patrizio. Analysis and control of linear switched system. Lecture notes. Politecnico Di Milano, 2009.

[14] Horváth Zsolt, Edelmayer András. Robust Model-Based Detection of Faults in the Air Path of Diesel Engines. Acta Universitatis Sapientiae Electrical and Mechanical Engineering. Vol.7, 2015, pp. 5-22.

[15] Edelmayer András, Bokor József, Keviczky László. An Ho Filtering Approach to Robust Detection of Failures in Dynamical Systems. In Proc. 33th Annual Decision and Control, Conf. Buena Vista, USA, 1994, pp. 3037-3039.

[16] Edelmayer András, Bokor József, Keviczky László. An Hœ
Filter Design for Linear Systems: Comparison of two Approaches. IFAC 13th Triennial World Congress. San Francisco, USA, 1996.

[17] Edelmayer András. Fault detection in dynamic systems: From state estimation to direct input reconstruction. Universitas-Gy őr Nonprofit Kft. Győr, 2012.

[18] Horváth Zsolt, Edelmayer András. Solving of the Modified Filter Algebraic Riccati Equation for H-infinity fault detection filtering. Acta Universitatis Sapientiae Electrical and Mechanical Engineering. Vol. 9, 2017, pp. 57-77.

[19] Geromel Jose C., Colaneri Patrizio. Ho and Dwell Time Specifications of Switched Linear Systems. Proceedings of the 47th IEEE Conference on Decision and Control. Cancun, 2008 .

[20] See supplementary material at http://home.deib.polimi.it/prandini/file/2015_06_16\%20hybri d\%20systems_2.pdf.

[21] Chen Weitian, Saif Mehrdad. Observer design for linear switched control systems. American Control Conference Proceedings of the 2004. 0-7803-8335-4, Boston, 2004.

[22] Horváth Zsolt, Csomós Petra. A Switched Linear System Approach to the Modeling of the Air Path of Diesel Engines. Proc. of the 14th ICCMSE International Conference of Computational Methods in Sciences and Engineering. Thessaloniki, 2018.

[23] See supplementary material https://de.mathworks.com/help/robust/ref/mincx.html.

at

[24] Gahinet Pascal, Nemirovski Arkadi, Laub Alan J. and Chilali Mahmoud. LMI Control Toolbox for Use with Matlab. The MathWorks Inc. Natick, 1995.

[25] Boyd Stephen, Laurent El Ghaoui, Feron Erik and Balakrishnan Venkataramanan. Linear Matrix Inequalities in System and Control Theory. SIAM. Philadelphia, 1994.

[26] See supplementary material at https://de.mathworks.com/help/robust/ref/feasp.html.

[27] Guang-Ren Duan, Hai-Hua Yu. LMIs in Control Systems: Analysis, Design and Applications. CRC Press. Boca Raton, 2013.

[28] Horváth Zsolt, Edelmayer András. An algorithm for the calculation of the dwell time constraint for switched H-infinity filters. Acta Universitatis Sapientiae, Electrical and Mechanical Engineering. Vol. 10, 2018, pp. 57-77.

[29] Horváth Zsolt, Edelmayer András. Determining the common minimum dwell time for switched $\mathrm{H} \infty$ fault detection filtering. Przeglad Elektrotechniczny. 2018.

[30] Jankovic Mrdjan, Kolmanovsky Ilya. Robust Nonlinear Controller for Turbocharged Diesel Engines. Proceedings of the American Control Conference. Philadelphia, PA, 1998.

[31] Horváth Zsolt, Edelmayer András. LTI-modelling of the Air Path of Turbocharged Diesel Engine for Fault Detection and Isolation. Invited paper in: Mechanical Engineering Letters. Vol. 14, ISSN 2060-3789, Gödöllő, 2016, pp. 172-188. 